\title{
Transatlantica
}

Revue d'études américaines. American Studies Journal

1 | 2017

Morphing Bodies: Strategies of Embodiment in

Contemporary US Cultural Practices

\section{Emeline Jouve, Susan Glaspell's Poetics and Politics of Rebellion}

Julie Vatain-Corfdir

\section{CpenEdition}

Journals

Édition électronique

URL : https://journals.openedition.org/transatlantica/9261

DOI : 10.4000/transatlantica.9261

ISSN : 1765-2766

Éditeur

Association française d'Etudes Américaines (AFEA)

Référence électronique

Julie Vatain-Corfdir, "Emeline Jouve, Susan Glaspell's Poetics and Politics of Rebellion », Transatlantica

[En ligne], 1 | 2017, mis en ligne le 16 octobre 2018, consulté le 24 mai 2021. URL : http://

journals.openedition.org/transatlantica/9261; DOI : https://doi.org/10.4000/transatlantica.9261

Ce document a été généré automatiquement le 24 mai 2021.

\section{cC)}

Transatlantica - Revue d'études américaines est mise à disposition selon les termes de la licence

Creative Commons Attribution - Pas d'Utilisation Commerciale - Pas de Modification 4.0 International. 


\title{
Emeline Jouve, Susan Glaspell's Poetics and Politics of Rebellion
}

\author{
Julie Vatain-Corfdir
}

\section{RÉFÉRENCE}

Emeline Jouve, Susan Glaspell's Poetics and Politics of Rebellion, Iowa City: University of Iowa Press, Studies in Theatre History and Culture, 2017, 258 pages, ISBN 978-1-60938-508-8 (pbk) / ISBN 978-1-60938-509-5 (ebk), 65 \$

1 Il est grand temps de réévaluer l'apport littéraire et théâtral des femmes qui écrivent, et ont écrit, pour la scène américaine. À l'heure où la critique apporte des nuances à la " grande histoire » du théâtre américain, trop souvent résumée à celle d'un réalisme au masculin, il s'avère fructueux de relire les autrices à travers d'autres prismes que ceux de l'histoire culturelle ou d'un féminisme qui court le risque d'abréger l'œuvre à l'une de ses dimensions. C'est l'une des raisons, parmi beaucoup d'autres, pour lesquelles le récent ouvrage d'Emeline Jouve, dédié au théâtre de Susan Glaspell dans toute sa poétique rebelle, est si bienvenu.

\section{Un corpus purement théâtral}

Publié - avec beaucoup d'à-propos - aux Presses Universitaires de l'Iowa, état natif de la fondatrice des Provincetown Players, Susan Glaspell's Poetics and Politics of Rebellion prend le parti de se consacrer entièrement à l'œuvre théâtrale de Susan Glaspell, ne convoquant son œuvre fictionnelle ou biographique qu'occasionnellement, pour éclairer l'analyse des pièces. Emeline Jouve écarte également de son étude les pièces écrites à quatre mains, préférant traquer l'esthétique de Glaspell à travers les treize pièces écrites par elle seule - pièces en un ou plusieurs actes, ayant fait l'objet de mises en scènes ou non. Le corpus s'étend donc de son premier succès devenu classique américain, Trifles (1916) jusqu'à sa pièce récompensée par le prix Pulitzer, Alison's House 
(1930), en passant par The People (1917), Close the Book (1917), The Outside (1917), Woman's Honor (1918), Bernice (1919), Inheritors (1922), The Verge (1921) et Chains of Dew (1922). Chose plus rare, l'analyse se penche également sur une œuvre récemment découverte (Free Laughter, 1919 ?), une pièce non jouée (Springs Eternal, 1943), et des fragments de pièce issus des archives, qui sont ici mis au jour et étudiés pour la première fois (Wings, date inconnue).

3 Jouve convoque une bibliographie critique sur Glaspell fournie (notamment Ben-Zvi, Gainor, Ozieblo, Hernando-Real, Carpentier, Noe), aux côtés de références plus attendues sur le théâtre américain et l'écriture au féminin. Elle place volontiers son analyse en dialogue avec la théorie critique européenne qui vient régulièrement éclairer - de manière ponctuelle plutôt que comme fil conducteur de tout un raisonnement - l'étude des pièces (Irigaray, Bergson, Barthes, Foucault, Bakhtine, Ricœur).

\section{La révolte comme mot d'ordre}

4 Reconnue pour sa plume de son vivant, Susan Glaspell a rapidement été confinée par l'histoire à un rôle d'adjuvante dont la vertu était d'avoir encouragé la création des pièces d'Eugene O'Neill au sein des Provincetown Players, cette aventure théâtrale dédiée aux dramaturgies américaines novatrices que Glaspell a conduite avec son mari, George Cram Cook, dès 1915. Ce n'est que dans les dernières décennies du $\mathrm{XX}^{\mathrm{e}}$ siècle que la critique a entamé une remise à l'honneur de Glaspell en tant qu'autrice, célébrant en elle la première dramaturge féministe américaine. Emeline Jouve offre ici, avec beaucoup de justesse, un troisième angle d'approche qui, tout en dialoguant avec les deux premiers, s'ancre dans le détail des répliques et des didascalies pour analyser de front, d'un bout à l'autre de l'œuvre, la poétique du texte et sa portée politique, toutes deux parallèlement placées sous le signe de la révolte. Jouve convoque ainsi en exergue de son étude l'analyse de Robert Brustein dans The Drama of Revolt: Studies in Modern Drama from Ibsen to Genet (1962). Brustein distingue le théâtre du passé, qu'il nomme celui de la communion, du théâtre moderne caractérisé par une attitude rebelle héritée du romantisme, dans un monde dominé par l'accident et la confusion. Ce clivage incite Jouve à proposer la révolte, le rejet des structures et des modes établis, comme clé de lecture de toute l'œuvre de Glaspell, dans sa forme comme dans son fond, d'abord dans la continuité des tendances définies par Brustein, puis au-delà, dans une réappropriation de la notion de révolte comme énergie positive, qui engage Glaspell à exploiter le pouvoir performatif de la littérature.

\section{Dénonciation, résistance, espoir}

5 L'ouvrage s'organise en neuf chapitres, regroupés en trois grandes parties. Chaque chapitre traite d'une ou plusieurs pièces (généralement deux ou trois) selon une approche thématique qui, si elle a le défaut d'émietter parfois l'analyse d'une œuvre entre plusieurs sections au risque de provoquer quelques redites ou de dérouter occasionnellement un lecteur moins versé dans l'œuvre de Glaspell, offre l'avantage d'une étude comparative convaincante. 
6 La première partie décline la notion de révolte en dénonciation de l'hypocrisie politique et du déclin des valeurs démocratiques américaines. Trifles, la plus connue et la plus glosée des pièces de Glaspell, condamne ainsi un système empreint de fausseté (" unjust justice ", 25) tout en rendant, grâce à une forme théâtrale guidée par la déduction policière, le pouvoir aux spectateurs devenus jury. Dès ce premier cas d'étude, Jouve révèle également le rapport problématique du personnage féminin à sa maison, rapport qui trahit les structures patriarcales de la société américaine, et que Glaspell représente au fil de toute son œuvre. Par l'absence comme par la dénonciation parodique, la dramaturge met en scène l'invisibilité de la femme dans la sphère domestique, où elle est partagée entre envie d'appartenance et besoin d'émancipation, ce qui inspire à Jouve un parallèle adroit avec l'œuvre d'Ibsen. Les procédés du comique, du pastiche (Woman's Honor) à l'humour et à la parodie (dans les pièces que Glaspell intitule "comédies») ou encore aux stéréotypes (Free Laughter) sont par ailleurs mis en avant comme moyens théâtraux efficaces pour dénoncer le poids de la tradition et les atteintes à la liberté d'expression.

7 Avec la deuxième partie, la dénonciation fait place à la résistance. Jouve s'y penche sur les personnages rebelles de l'œuvre de Glaspell, distinguant les révoltes idéalistes telle celle de Madeline, Antigone moderne luttant pour la justice dans Inheritors - des révoltes individualistes, liées à la trajectoire du personnage, qui choisit de vivre en marge de la collectivité (The Outside) ou de lui tenir tête en rejetant tout ce qu'elle lui impose (The Verge). Les rebelles de Glaspell invitent Jouve à conjuguer au féminin le portrait de l'homme révolté proposé par Camus, et lui inspirent de belles pages d'analyse formelle, notamment à propos des rythmes du texte et de la symbolique spatiale. L'étude des didascalies d'ouverture éclaire ainsi la dimension métaphorique d'espaces scéniques où chaque élément est révélateur de sens - jusqu'au sel, qui lie la mer à la terre et corrode l'existence (Wings) - et met en relief l'ambivalence du décor expressionniste de The Verge, où l'intertexte platonicien invite le spectateur à rechercher l'idée, au-delà du visuel.

8 La troisième partie défend le théâtre de Glaspell comme une dramaturgie de l'espoir, au-delà des tendances plus pessimistes décrites par Brustein. Jouve met ainsi en lumière, au fil des pièces, le trait peut-être le plus distinctif de Glaspell en tant que dramaturge moderne : son plaidoyer pour la collaboration, conséquence positive de la révolte. Cette collaboration prend d'abord le visage de la solidarité féminine («sisterhood », 169) dans Trifles et The Outside, avant de revêtir les traits de la solidarité nationale dans Inheritors et Springs Eternal. Moins convaincant, le dernier volet de cette partie propose le dévouement, voire le sacrifice ("self-sacrifice", 203), comme modalité ultime et paradoxale de la révolte féminine. Le parallèle avec le mimétisme irigarayien comme mode subversif de réappropriation du féminin, s'il soulève une question intéressante, aurait mérité, pour être plus probant, une analyse plus approfondie des modalités parodiques du dialogue et de la construction dramatique dans les pièces étudiées, Bernice et Chains of Dew.

9 L'étude d'Emeline Jouve est précise, et ancrée dans la prise en compte fine du contexte historique, politique et littéraire de son auteur. Elle s'attache à déceler les idées politiques de Glaspell dans tous les aspects de son théatre : la cadence des mots comme l'intensité de l'éclairage, interrogeant les notions de réalisme et d'expressionnisme qui travaillent la création de l'époque. Certaines clés d'analyse reviennent peut-être un peu souvent - nombreuses sont par exemples les métaphores « reprises » ou « littéralisées » 
- et l'on peut regretter par ailleurs que davantage de dramaturges américains ne soient pas convoqués parallèlement, pour enrichir la discussion et définir l'originalité de Glaspell autant que son influence (Thornton Wilder au sujet de la pantomime de Inheritors, par exemple). Ceci n'enlève rien au fait que Susan Glaspell's Politics and Poetics of Rebellion est une contribution précieuse aux études théâtrales américaines, qui dresse le portrait rafraîchissant et convaincant d'une autrice innovante, à jamais insoumise.

INDEX

Thèmes : Recensions

\section{AUTEURS}

JULIE VATAIN-CORFDIR

Sorbonne Université 